\title{
Cloud System Design using AMQP Protocol for Smart Devices System Applications
}

\author{
Trio Adiono ${ }^{1}$, Rahmat Muttaqin ${ }^{1}$, Renitia Murti Rahayu ${ }^{1}$, Rachmad Vidya Wicaksana Putra ${ }^{1}$ \\ ${ }^{\prime}$ Microelectronics Center, Institut Teknologi Bandung, Indonesia \\ Email: tadiono@stei.itb.ac.id
}

\begin{abstract}
In this publication, we propose a cloud system design for internet-of-things (IoT) based smart devices system using $A M Q P$ protocol and RabbitMQ server. Using this proposed system, every connected device in smart devices system can be controlled remotely by mobile user, as long as internet connection is provided. Furthermore, if the internet connection between cloud and smart devices system is unstable or even off in a while, the cloud system is able to save the data to RabbitMQ server and send them when the internet connection stable again. In order to receive and store the data sent by user or smart devices system's gateway, the cloud system utilizes exchange which is created in the server. If the data have reached the server, they will be stored and passed to exchange for next transmission. These data are bound with certain queue in order to organize the transmission schedule. By using such mechanism, the proposed system can overcome the internet connection problem without losing any data and provide a reliable cloud system.
\end{abstract}

Keywords: Cloud system, internet-of-things, AMQP, RabbitMQ, smart devices system

\section{Introduction}

Currently, Internet-of-Things (IoT) technology is emerging as a main enabler for many applications in the world. It is proven by machine-to-machine communication (M2M) between two or more devices via internet connection. These devices interact with others in one global internet network using specific protocol and communication scheme. They also do data processing based on specific application.

One of its application that draws massive attention is smart home system. Regarding this topic, we have explored its potentials through researches and experiments starting from protocol, modules, application and system level [1]-[7]. We have successfully designed a smart home system and expanded its potentials for more general purpose applications called smart devices system (smart devices system). Using this general purpose concept, hopefully the applications can be more various instead of only on smart home.

In this system, we proposed an integrated scheme that includes indoor and outdoor environments. For indoor, the system uses a wireless sensor network (WSN) scheme, while for outdoor, it uses internet cloud scheme. Previous researches have discussed a lot mainly on indoor environment, thus this research aims to discuss on the outdoor environments (i.e. cloud systems). Figure 1 shows the position of cloud system that we investigated.

In Figure 1, we see that the position of cloud system is very strategic in connecting user and smart devices system. One of the control scenario can be conducted by transmitting data from user to smart devices system (e.g. smart home). User send data to the cloud system via internet connection (e.g mobile data). Afterwards, the cloud system holds the data from user and transmit them to the destination device. Data received by the target device are processed to provide a proper response depends on the command within. Trail of response is in a reverse order and it is processed gradually from devices, host, gateway, cloud system until back to user again. In this communication system, we can see that one of the crucial part is performed by cloud system. Hence, a reliable cloud system is needed.

In a reliable system, the data exchange process needs an efficient server provider. The data storage can be accessed from anywhere, even from solitary place as long as there is an internet connection. Furthermore, we need a cloud system that is capable of overcoming disruption, unstable and uneven internet connection, since it still happens sometimes

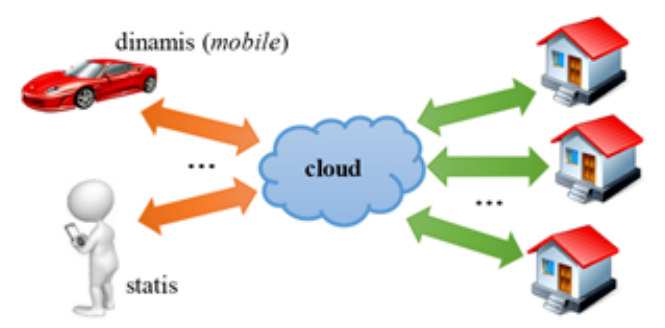

Figure 1. Illustration of Cloud System Position in IoT Environment. 
especially in rural area. These are the research questions for this work.

In order to fulfill the requirements, we choose and design protocol and broker system that are capable to provide messaging service via internet connection. Furthermore, the broker must arrange the transaction data using a specific protocol and handle the problem that may arise from unstable connection. In this research, we choose Advanced Message Queuing Protocol (AMQP) protocol and RabbitMQ broker. AMQP is considered more compatible with unstable internet condition because it has store-and-forward feature function. This feature can save data temporarily if internet connection unstable. AMQP also provides high interoperability (i.e. multi-user, multi-infrastructure, flexible-routing) and security. Furthermore, AMQP has configurable and complete superset library. To run AMQP protocol, we utilize RabbitMQ broker. Using these approaches, we design cloud system that can cope the requirements and obtain the desired cloud system.

This research article is presented in a number of sections. First part is background of the research, second part is research methodology and third part is cloud system design. Then it is followed by result, analysis and conclusions respectively.

\section{Research Methodology}

\subsection{Overview}

This research is continuation from previous researches on smart home system [1]-[7], in which we expand its perspective into a general smart devices system and focus the discussion on the outdoor environment (i.e. cloud system).

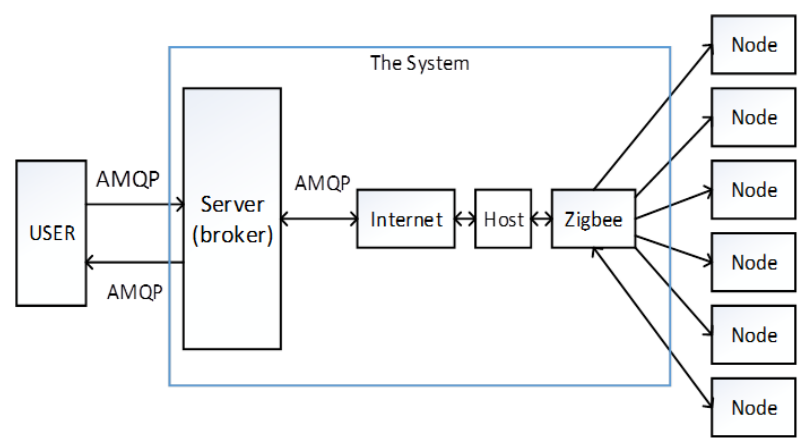

Figure 2. Proposed Cloud System.

Figure 2 shows that the proposed smart devices system consists of user, server, host and node (devices). User can utilize devices such as smart phone for sending or receiving data to or from server. Server is utilized to collect data from user and host. To run this system, we need a fast and lightweight protocol that have good performance and security. Choosing a protocol affect in choosing a server as well. Furthermore, on indoor side, host is used to receive and send data from and to server, as well as, from and to node. Therefore, we need a host that is flexible, high-interoperability and easy to configure. Last part is node which acts corresponding to the command from host.

\subsection{Protocol and Broker}

There are some protocols that can be used to perform M2M communication such as AMQP, HTTP, MQTT, STOMP, CoAP, etc. The popular protocols for IoT based applications are Hypertext Transfer Protocol (HTTP), Message Queue Telemetry Transport (MQTT) and Advanced Message Queuing Protocol (AMQP) because of their functionalities and robustness. The differences between HTTP, MQTT and AMQP are presented in Table 1, comprising type, speed, function, protocol, synchronization and queue. These points are used as consideration in choosing the protocol. The priorities are focused on speed and high interoperability (i.e. multi-user and multi infrastructure features).

Table 1. Comparison HTTP, MQTT and AMQP

\begin{tabular}{llll}
\hline & HTTP & MQTT & AMQP \\
\hline Type & $\begin{array}{l}\text { Request } \\
\text { and }\end{array}$ & $\begin{array}{l}\text { Publish } \\
\text { and }\end{array}$ & $\begin{array}{l}\text { Publish } \\
\text { and }\end{array}$ \\
& Respond & Subscribe & Subscribe \\
Speed & Slow & Fast & Fast \\
Function & General & Specific & Specific \\
Protocol & TCP/IP & TCP/IP & TCP/IP \\
Synchron & Synchron & Asynchron & Asynchron \\
Queue & Yes & No & No \\
\hline
\end{tabular}

From Table 1, the HTTP is eliminated because it has slower speed than others. This behavior is caused by request and synchronization process which need a significant running time. This can be exacerbated by interoperability requirement. Quite different to HTTP, MQTT is designed as lightweight broker protocol based on small publishand-subscribe messaging for small footprint code, low bandwidth and low power [8]. MQTT is suitable for modules which need low bandwidth and power, such as node devices in embedded modules. However, in order to be able to do such diverse and various communication scheme for highinteroperability function, MQTT is inadequate.

Different to previous protocols, AMQP is designed to support messaging for almost every business aplication and using open source library [9]. AMQP is also used as standard for instant messaging. AMQP protocol is considered more compatible with unstable internet connection because it has store-and-forward feature. This feature can save data temporarily if connection unstable or even off in a while. AMQP also has a good interoperability, because it can provide 
multi-user, multi-infrastructure, flexible-routing and security. Furthermore, AMQP is configurable and supported with a complete superset library. Therefore, we choose AMQP protocol as a basic protocol for the proposed cloud system. In order to run AMQP protocol, we use RabbitMQ broker.

\section{Cloud System Design}

\subsection{Protocol}

Protocol used for communication between server and host and server and user is AMQP 0-9-1. For implementation, python $2.6-3.3$ is used as programming language and pika 0.10 .0 as library. RabbitMQ is utilized as broker that performs data transaction based on the AMQP 0-9-1 protocol.

\subsection{Server}

The selected server is RabbitMQ because of its compatibility to AMQP protocol. RabbitMQ is a provider of data transmission service, commonly referred as broker. On the server side, there are exchange and queue. Exchange is used to receive incoming data from publisher while the queue is used to accumulate data for transmission to subscriber. Exchange can be declared on sender, receiver, or directly on the RabbitMQ server.

Incoming data from the publisher are sent to the exchange and the publisher does not even know if their data are going to be sent by the exchange (server) or not. There are two types of exchange, namely direct and fan-out (broadcast). The direct type sends data to queue through the exchange in accordance with its routing-key. Afterwards, queue binds itself to the exchange in which the data are connected to. Illustration of this mechanism is shown on Figure 3. If exchange publishes the data with routing key "conA", then Queue-B that binds itself to the exchange with routing key "conB", is disable to receive data. The one that is enabled to receive data is Queue-A because it has routing key "conA".

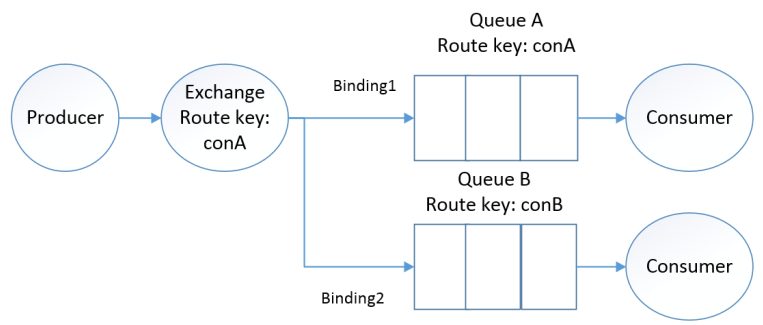

Figure 3. Direct Exchange

Quiet different to the dirct type, the fan-out type sends data to all queue through exchange without using routing key. Data can be stacked in the queue and distributed to receiver alternately depends on how many receiver that are connected to the queue.
Hence, the fan-out type is similar with broadcast. Illustration of this mechanism is shown in Figure 4.

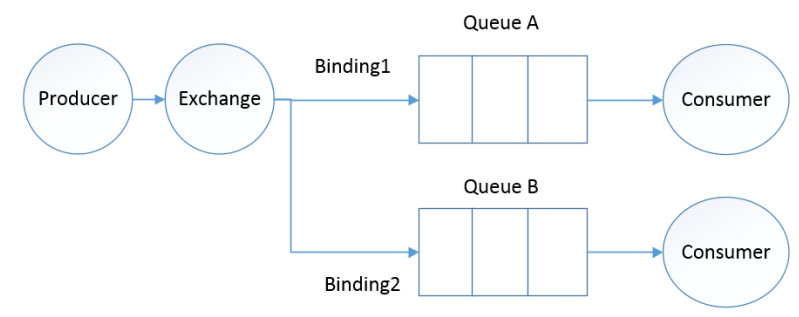

Figure 4. Fan-out Exchange

RabbitMQ server uses PC with Linux Operating System (OS). On this server, we create user, password, virtual host, queue and exchange. These queue and exchange can be created by host programming or set in server's Graphical User Interface (GUI). There are two types of exchange used here (i.e. 2 direct and 2 fan-out). Our proposed server design is to utilize a direct excange for incoming data and fan-out exchange for output data. Queue is utilized by host and user only if they want to request data from server. Thus, we create 4 exchanges in order to avoid interference among the data. This scheme enables a fast reception and transmission for any mode (i.e. control and monitor). Illustration of this mechanism is shown in Figure 5.

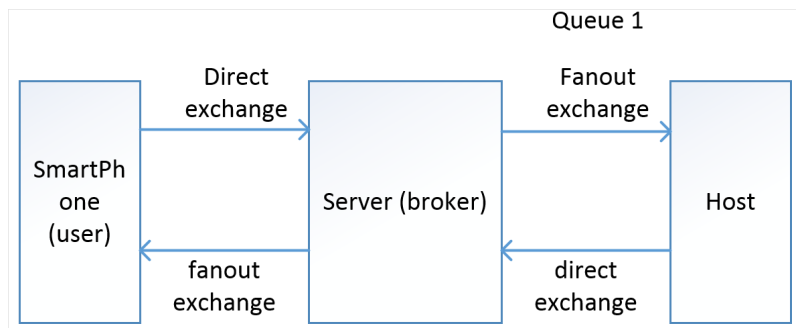

Figure 5. Position of Exchange and Queue on Server

\subsection{Host}

Host is used as a regulator of data that come from server and application node devices. The accepted data from server have JSON format and they are decoded in the host to control the application nodes such as switch, RGB lamp, door lock, fan, curtain, etc. For command function, host only needs to receive data from server, processes them according to the format and then sends them to the application or target node. Afterwards, node acts according to the command within the received data. For monitoring function (e.g. temperature and humidity), the process is similar with the command function but the difference is that the node gives the response and send the data back to the host. Thus, the host needs to receive the data and process them 
into JSON formatted data and send them back to the server.

Host is essentially a mini-PC equipped with wireless communication module (i.e ZigBee) and connected to internet. The flowchart of host programming is presented in Figure 6. Point A represents the specific processing for each target device (node), for example: RGB lamp, door lock, fan control, curtain control, humidity, temperature, etc. Thus from this point A, there are branches of programming for specific application which are not discussed thoroughly here. If each target application is executed, the program run to point $B$ representing a loop process.

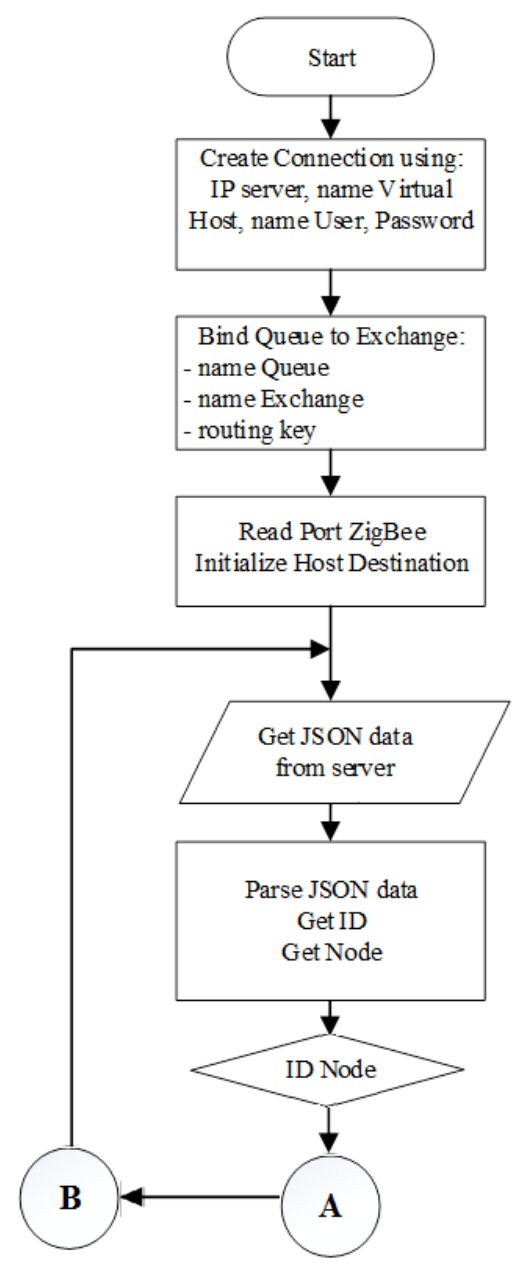

Figure 6. Flowchart Host

At start, host is connected to server using AMQP protocol, hence we have to know the IP address of the server, virtual host name, username and password to access RabbitMQ server. Host connects to the queue of the server which has been bound to the exchange with fan-out type. Host receives the JSON formatted data from server and decode them to get ID value, node's name and data payload. If messages are successfully decoded, host sends them to the target node using ZigBee protocol.
For control mode (command function), host receive data from server, processes them according to the JSON format and then sends them to the target node. Afterwards, node acts according to the command within the received data. For monitoring mode, the process is similar with the control mode but the node gives the response back. It processes the command and sends the data back to the host. Thus, the host receives the data and translate them into JSON format and send them back to the server. The user can access the result if the data have reached the server using internet-connected devices, such as smart phone, pad, etc.

\section{Results and Discussion}

In order to verify the proposed cloud system's functionality, mobile application based testing scenario using smart phone is conducted. If we send a JSON formatted data from smart phone to server, then the data are captured in the server and passed to host of the smart devices system. If the server is built properly, it would be capable on delivering the received data to host. Figure 7 shows JSON formatted data that are delivered from mobile application to server and received by host. It proves that the server (cloud) system is working properly, delivering the command data to the host.

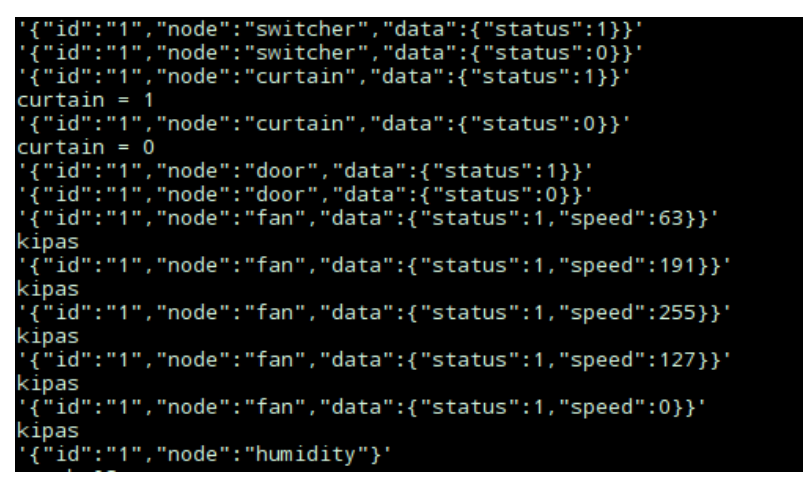

Figure 7. JSON Data Received by Host

Important parameters in JSON formatted data are ID, node's name, and data. For this testing, all ID is set by 1 since there is only one target device (node) controlled. Meanwhile node's name of the device is set in a form of bitstream. For example, door loock node is named : "door" with ID : " 1 ". It means that door lock node is the first device. Thus, if its payload data has value " 1 ", then the door is opened and otherwise ("0"), it is closed. For fan control "fan", there is a variable named "speed" that represents the speed of the fan itself. Meanwhile, for temperature and humidity monitor, the received data only consist of ID and node's name, but the corresponding nodes need to response and answer the command with the measured temperature and humidity (Figure 8). From Figure 8, we can see that JSON formatted data sent by host to cloud system contain the measured 
temperature and humidity values, namely 32 degree of Celcius for temperature and $36 \%$ for humidity.

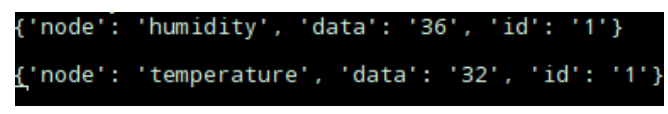

Figure 8. Data JSON Sent from Host to Cloud

Host may not be able to receive any data from cloud if the internet connection between cloud and host is off. Since the proposed system has store-andforward feature, the data that have reached server but not sent to host yet, are saved temporarily in RabbitMQ server. These data are still in idle status and gethering in an idle storage as shown in Figure 9. If the internet connection is stable or on again, server sends these all data to host immediately.

\begin{tabular}{|c|c|r|r|r|r|}
\hline \multicolumn{3}{|c|}{ Overview } & \multicolumn{3}{c|}{ Messages } \\
\hline Name & Features & State & \multicolumn{1}{|r|}{ Ready } & Unacked & \multicolumn{1}{c|}{ Total } \\
\hline Name-1 & Excl AD & idle & 0 & 0 & 0 \\
\hline Name-2 & $\underline{\text { D }}$ & idle & 1 & 0 & 1 \\
\hline Name-3 & \multicolumn{1}{c}{} & idle & 0 & 0 & 0 \\
\hline
\end{tabular}

Figure 9. Temporary Data Storage in RabbitMQ Server

\section{Conclusion}

The proposed cloud system design using AMQP protocol and RabbitMQ server is working properly. This proposed cloud system has a good interoperability because it supports multi-user and multi-infrastructure integration. Furthermore, the proposed system can overcome the unstable internet connection problem using store-and-forward feature. Hence, if internet connection is unstable or even off for a while, data are saved temporarily in the server until the internet connection being normal again. The host can also connect to the cloud system again immediately according to final state. These are proofs that the proposed system is potentially utilized for broad applications on IoT based smart devices systems.

\section{References}

[1] T. Adiono, R. V. W. Putra, M. Y. Fathany, M. A. Wibisono and W. Adijarto, Smart Home Platform based on Optimized Wireless Sensor Network Protocol and Scalable Architecture, "Proc. of 2015 9th International Conference on Telecommunication Systems Services and Applications (TSSA)", November 2015, pp. 1-5.

[2] T. Adiono, R. V. W. Putra, M. Y. Fathany, and W. Adijarto, Desain Sistem Rumah Cerdas Berbasis Topologi Mesh dan Protokol Wireless Sensor Network yang Efisien, "Jurnal Informatika,
Sistem Kendali, dan Komputer (INKOM)", Vol. 9, No. 2, November 2015, pp. 65-72.

[3] B. L. Lawu, M. Y. Fathany, K. Afifah, M. H. Santriaji, R. V. W. Putra, S. Fuada, and T. Adiono, Prototyping Design of Mechanical Based End-Devices for Smart Home Applications, "Proc. of 2016 4th Int. Conf. on Information and Communication Technology (ICoICT)", May 2016, pp. 1-6.

[4] T. Adiono, R. V. W. Putra, M. Y. Fathany, K. Afifah, M. H. Santriaji, B. L. Lawu, and S. Fuada, Prototyping Design of Electronic End-Devices for Smart Home Applications, "Proc. of 2016 IEEE Region 10 Symposium (TENSYMP)", May 2016, pp. 261-265.

[5] T. Adiono, R. V. W. Putra, M. Y. Fathany, B. L. Lawu, K. Afifah, M. H. Santriaji, and S. Fuada, Rapid Prototyping Methodology of Lightweight Electronic Drivers for Smart Home Appliances, "Int. J. of Electrical and Computer Engineering (IJECE)", Vol. 6, No. 5, October 2016, pp. 21142124.

[6] T. Adiono, M. Y. Fathany, R. V. W. Putra, K. Afifah, M. H. Santriaji, B. L. Lawu, and S. Fuada, Live Demonstration: MINDS Meshed and Internet Networked Devices System for Smart Home: Track Selection: Embedded Systems, "Proc. of 2016 13th IEEE Asia Pacific Conf. on Circuits and Systems (APCCAS)", October 2016, pp. 736-737.

[7] K. Afifah, S. Fuada, R. V. W. Putra, M. Y. Fathany, and T. Adiono, Design of Low Power Mobile Application for Smart Home, "Proc. of 2016 IEEE Int. Symp. on Electronics and Smart Devices (ISESD)", November 2016, pp. 1-5.

[8] K. Grgic and I. S. I. Hedi, A Web-Based IoT Solution for Monitoring Data Using MQTT Protocol, "Proc. of Int. Conf. on Smart Systems and Technologies (SST)", October 2016, pp. 249-253.

[9] P. Peniak and M. Franekova, model of Integration of Embedded Systems via CoAP Protocol of Internet of Things, "Proc. of 2016 Int. Conf. on Applied Electronics (AE)," September 2016, pp. 201-204. 